\title{
Short communication: 3-Phenyllactic acid production in milk by Pediococcus pentosaceus SK25 during laboratory fermentation process
}

\author{
Shuhuai Yu, ${ }^{*}$ Chen Zhou, ${ }^{*}$ Tao Zhang, ${ }^{*}$ Bo Jiang, ${ }^{*} \dagger$ and Wanmeng Mu$^{*}{ }^{1}$ \\ *State Key Laboratory of Food Science and Technology, Ministry of Education, Key Laboratory of Carbohydrate Chemistry and Biotechnology, and \\ †Synergetic Innovation Center of Food Safety and Nutrition, Jiangnan University, Wuxi 214122, China
}

\begin{abstract}
3-Phenyllactic acid (PLA) is a broad-spectrum antimicrobial compound, produced by a wide range of lactic acid bacteria. A novel lactic acid bacteria strain with high PLA-producing ability, Pediococcus pentosaceus SK25, was isolated from traditional Chinese pickles. When grown in de Man, Rogosa, Sharpe broth at $30^{\circ} \mathrm{C}$ for $36 \mathrm{~h}$, this strain produced $135.6 \mathrm{mg} / \mathrm{L}$ of PLA. Using this strain as starter for milk fermentation, 47.2 $\mathrm{mg} / \mathrm{L}$ of PLA was produced after fermentation for 12 $\mathrm{h}$. The PLA production was significantly improved by phenylalanine supplement, but was completely inhibited by tyrosine supplement.
\end{abstract}

Key words: 3-phenyllactic acid, lactic acid bacteria, fermentation, Pediococcus pentosaceus

\section{Short Communication}

3-Phenyllactic acid (PLA) is an organic acid with broad-spectrum antimicrobial activity (Mu et al., $2012 b)$. It inhibits not only food-borne pathogenic bacteria, including Listeria monocytogenes (Dieuleveux and Gueguen, 1998), Staphylococcus aureus, and Escherichia coli O157:H7 (Ohhira et al., 2004), but also food-spoiling fungi, including yeasts such as Candida pulcherrima, Candida parapsilosis, and Rhodotorula mucilaginosa (Schwenninger et al., 2008), and a wide range of molds, such as Aspergillus ochraceus, Penicillium roqueforti, and Penicillium citrinu (Lavermicocca et al., 2003). Due to its broad inhibitory activity against a variety of food-borne microorganisms, PLA has interesting potential as an antimicrobial agent in the food industry.

3-Phenyllactic acid is a phenolic acid commonly found in honey, and its content is commonly much higher than that of other phenolic acids in honey. It has even been suggested as a chemical marker for some honeys (Tuberoso et al., 2011). Recently, PLA was proven to be a regular metabolite from lactic acid bac-

Received July 21, 2014.

Accepted October 16, 2014

${ }^{1}$ Corresponding author: wmmu@jiangnan.edu.cn teria $(\mathbf{L A B})$ and was produced by a wide range of $\mathrm{LAB}$ species (Valerio et al., 2004). Laboratory research has suggested that PLA could be produced in fermented foods using LAB as starter, such as sourdoughs (Van der Meulen, et al., 2007; Ryan et al., 2009). During the sourdough fermentation process, fungal growth was significantly delayed in the presence of PLA-producing LAB strain, Lactobacillus plantarum 21B, in coculture with Saccharomyces cerevisiae (Lavermicocca et al., 2000).

In our study, a novel LAB strain, Pediococcus pentosaceus SK25, was isolated from traditional Chinese pickles and its PLA-producing ability was measured in de Man, Rogosa, Sharpe (MRS) broth. We subsequently evaluated PLA production by this strain in milk through simulating the LAB fermentation manufacturing process, and we analyzed the effect of supplementation with AA precursors on PLA production during the fermentation process.

Pediococcus pentosaceus SK25 was isolated from traditional Chinese pickles and cultivated in MRS medium at $30^{\circ} \mathrm{C}$ without shaking. To obtain the LAB starter for milk fermentation, the overnight culture of P. pentosaceus SK25 was collected and centrifuged at 4,000 $\times g$ for $15 \mathrm{~min}$ at $4^{\circ} \mathrm{C}$. The pellet was then washed twice and resuspended in sterile $0.9 \%$ (wt/vol) $\mathrm{NaCl}$ to give a final concentration of approximately $5 \times 10^{9} \mathrm{cfu} / \mathrm{mL}$ for further inoculation of the milk fermentation process.

The PLA production by $P$. pentosaceus SK25 was tested in commercial homogenized milk. Commercial fresh milk (full-fat pasteurized milk, Mengniu Modern Meadow brand, product license No. QS10005010354; Huhehaote, Inner Mongolia, China) was purchased from a local supermarket. Every $100 \mathrm{~mL}$ of milk contained $3.4 \mathrm{~g}$ of protein, $4.0 \mathrm{~g}$ of fat, $5.1 \mathrm{~g}$ of carbohydrate, 66 mg of sodium, $118 \mathrm{mg}$ of calcium, and $292 \mathrm{~kJ}$ of energy. Milk samples $(100 \mathrm{~mL})$ supplemented with $2 \%$ glucose were inoculated with $P$. pentosaceus SK25 with initial dose of $10^{7} \mathrm{cfu} / \mathrm{mL}$. Milk fermentations were conducted at $30^{\circ} \mathrm{C}$ in a water bath for $12 \mathrm{~h}$ to simulate conditions of yogurt production. Starting after $6 \mathrm{~h}$ of incubation, the samples were withdrawn every $1 \mathrm{~h}$ for PLA quantification analysis. 
The fermented milk samples were centrifuged at 4,000 $\times g$ for $15 \mathrm{~min}$ at $4^{\circ} \mathrm{C}$. The supernatant was treated with TCA to remove the proteins by precipitation, followed by another round of centrifugation at 20,000 $\times g$ for $15 \mathrm{~min}$ at $4^{\circ} \mathrm{C}$. The ultimate supernatant was filtered $(0.22-\mu \mathrm{m}$ pore size membrane) for further PLA determination. The PLA was determined using an Agilent 1260 liquid chromatography system equipped with a 1260 Infinity Diode Array Detector (Santa Clara, $\mathrm{CA}$ ) and an Agilent Zorbax SB-C $\mathrm{C}_{18}$ column (4.6 $\mathrm{mm} \times$ $150 \mathrm{~mm}, 5 \mu \mathrm{m}$ ). Linear gradient elution was used with methanol $/ 0.05 \%$ trifluoroacetic acid (solvent $\mathrm{A}$ ) and water $/ 0.05 \%$ trifluoroacetic acid (solvent B) at $1 \mathrm{~mL} /$ min and A:B ratios of 10:90, 100:0, 100:0, and 10:90, with run times of $0,20,23$, and $25 \mathrm{~min}$, respectively. The PLA was detected by UV detection at $210 \mathrm{~nm}$.

The PLA-producing microorganisms were isolated from traditional Chinese pickles using MRS broth for cell culture and HPLC for detection. Forty-five strains were isolated that produced more than $0.1 \mathrm{~m} M$ PLA when grown in MRS broth at $30^{\circ} \mathrm{C}$ for $36 \mathrm{~h}$, and the strain SK25 displayed the highest PLA-producing ability of $0.81 \mathrm{~m} M(135.6 \mathrm{mg} / \mathrm{L}$; Figure 1$)$. The $16 \mathrm{~S}$ rDNA sequence of the strain SK25 displayed 100\% identity with that of $P$. pentosaceus DSM 20336 (GenBank accession No. NR_042058); thus, the screened strain was identified as $P$. pentosaceus. Pediococcus strains are widely distributed in fermented beverages and foods (Tanasupawat et al., 1993). In a previous study, Pediococcus acidilactici DSM 20284, which was isolated from barley (Tanasupawat et al., 1993), was reported to be able to produce $0.65 \mathrm{~m} M$ PLA in MRS broth after $36 \mathrm{~h}$ of fermentation (Mu et al., 2012a). The PLA is a possible metabolite of phenylalanine and could be produced by a wide range of LAB strains. But the PLA productivities of different LAB strains vary greatly. Shown in Table 1, most LAB strains produced less than $1 \mathrm{~m} M$ PLA when grown in MRS broth; however, Lactobacillus plantarum 1081 produced $5.2 \mathrm{mM}$ PLA (Gerez et al., 2010).

3-Phenyllactic acid is commonly found in honey; however, few reports of PLA in other commercial food products exist. Plenty of reports have shown that PLA could be produced by a wide range of LAB during microbial fermentation, so theoretically PLA may also be produced in LAB-fermented foods. We first determined the PLA content in commercial LAB-fermented yogurts. Four brands of LAB-fermented yogurts were selected for PLA detection and 3 samples were tested for each brand. The results showed that 2 did not contain

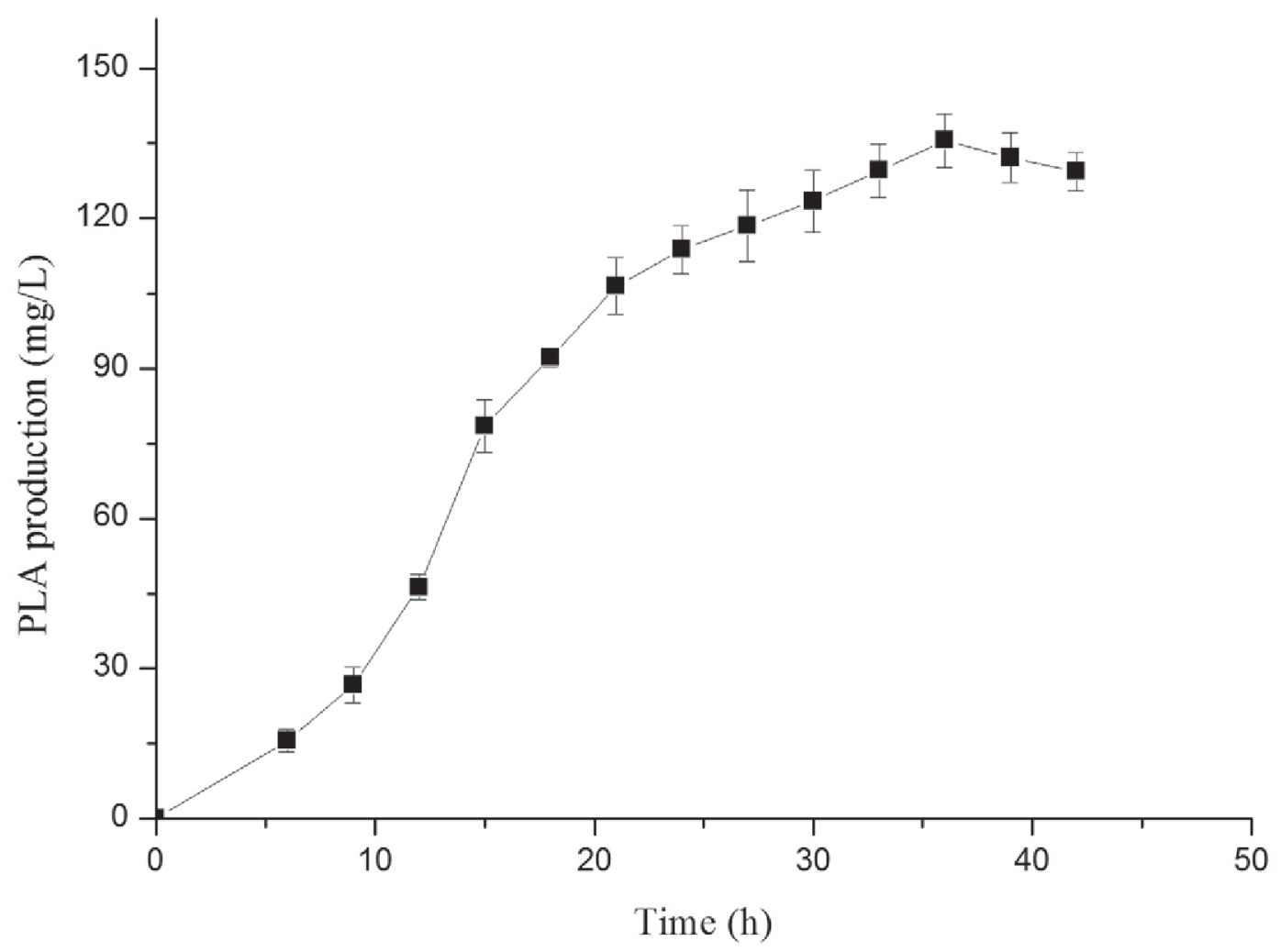

Figure 1. Production of 3-phenyllactic acid (PLA) during Pediococcus pentosaceus SK25 fermentation at $30^{\circ} \mathrm{C}$ in de Man, Rogosa, Sharpe broth. Values are means of 3 replications \pm SD. 
Table 1. 3-Phenyllactic acid (PLA) production by selected LAB strains grown in de Man, Rogosa, Sharpe broth

\begin{tabular}{|c|c|c|c|}
\hline Strain & Source & $\begin{array}{l}\text { PLA production } \\
(\mathrm{m} M)\end{array}$ & Reference \\
\hline Pediococcus pentosaceus SK25 & Traditional Chinese pickles & $0.81 \pm 0.06$ & This study \\
\hline Pediococcus acidilactici DSM 20284 & Barley & 0.65 & Mu et al., 2012a \\
\hline Lactobacillus plantarum CECT-221 & Spanish Collection of Type Cultures & 1.38 & Rodriguez et al., 2012 \\
\hline L. plantarum 1081 & $\begin{array}{l}\text { Culture Collection of the Centro de Referencia } \\
\text { para Lactobacilos }\end{array}$ & 5.2 & Gerez et al., 2010 \\
\hline Weissella confuse ITM14A & Sourdough & 0.06 & Valerio et al., 2004 \\
\hline Lactobacillus alimentarius ATCC 29643 & Fish products & 0.37 & Valerio et al., 2004 \\
\hline L. plantarum VE56 & Fermented cassava & 0.48 & Ndagano et al., 2011 \\
\hline Weissella cibaria FMF4B16 & Milk flour & 0.1 & Ndagano et al., 2011 \\
\hline
\end{tabular}

PLA, but the other 2 contained $13.2 \pm 0.2$ and $21.8 \pm$ $0.3 \mathrm{mg} / \mathrm{L}$ of PLA, respectively. The PLA content in the commercial LAB-fermented yogurts was similar to that in most honeys, which generally have less than $100 \mathrm{mg} /$ $\mathrm{kg}$ of PLA (Dimitrova et al., 2007).

We also tested the capacity of $P$. pentosaceus SK25 to produce PLA during milk fermentation at $30^{\circ} \mathrm{C}$. The PLA content was $17.0,23.7,29.1,34.8,39.3,43.5$, and $47.2 \mathrm{mg} / \mathrm{L}$ when P. pentosaceus SK25 was fermented in the milk for $6,7,8,9,10,11$, and $12 \mathrm{~h}$, respectively, showing that the PLA content gradually increased with longer fermentation times (Table 2). The total PLA production of milk fermentation using $P$. pentosaceus SK25 as a starter was higher than that of the 2 selected commercial LAB-fermented yogurt products in which PLA was detected. In previous studies, wheat sourdough fermentation was simulated using several types of PLA-producing LAB strains as starter, and it was found that 5 to $35 \mathrm{mg} / \mathrm{kg}$ of PLA could be produced after fermentation for $48 \mathrm{~h}$ (Ryan et al., 2009). Van der Meulen et al. (2007) analyzed the metabolite production during laboratory fermentations of wheat and spelt sourdoughs and found PLA in 35 out of 40 sourdough samples at concentrations usually lower than $0.3 \mathrm{mM}$ / $\mathrm{kg}(49.8 \mathrm{mg} / \mathrm{kg})$. Fermentation of quinoa and wheat slurries by L. plantarum CRL 778 could produce 45.5 and $17.5 \mathrm{mg} / \mathrm{L}$ of PLA, respectively (Dallagnol et al., 2013).

Use of antimicrobial agent-producing LAB strains as starter improves the antimicrobial activity of LABfermented food products. Lavermicocca et al. (2000) reported that wheat sourdough fermented by the PLAproducing LAB strain (L. plantarum 21B) in coculture with Saccharomyces cerevisiae had much longer shelf life $(>7$ d) than that fermented by only $S$. cerevisiae $(<2$ d). Pediocin is another antimicrobial compound with activity against several food-borne pathogens. Somkuti and Steinberg (2010) reported that in simulated yogurt fermentation, the pediocin-producing strain P. acidilactici F in coculture with Streptococcus thermophilus and Lactobacillus delbrueckii ssp. bulgaricus could effectively produce pediocin and showed inhibitory activity against Listeria monocytogenes. Our study showed that P. pentosaceus SK25 starter produced PLA when cultured in milk. The inhibitory activity of PLA-produced fermented milk against bacteria and fungi will be extensively investigated in the future.

Milk fermentation by $P$. pentosaceus SK25 was also studied under supplementation with $1 \mathrm{mg} / \mathrm{mL}$ of phenylalanine or tyrosine. The 2 AA supplements produced different results. The phenylalanine supplement significantly increased PLA production during the milk fer-

Table 2. 3-Phenyllactic acid (PLA) production (mg/L) in milk fermented with Pediococcus pentosaceus SK25 as starter

\begin{tabular}{lccc}
\hline Time $(\mathrm{h})$ & $\begin{array}{c}\text { Milk }+2 \% \\
\text { glucose }\end{array}$ & $\begin{array}{c}\text { Milk }+2 \% \text { glucose } \\
+1 \mathrm{mg} / \mathrm{mL} \text { of phenylalanine }\end{array}$ & $\begin{array}{c}\text { Milk }+2 \% \text { glucose } \\
+1 \mathrm{mg} / \mathrm{mL} \text { of tyrosine }\end{array}$ \\
\hline 6 & $17.0 \pm 3.2$ & $36.1 \pm 3.7$ & $\mathrm{ND}^{2}$ \\
7 & $23.7 \pm 3.5$ & $39.3 \pm 4.2$ & $\mathrm{ND}$ \\
8 & $29.1 \pm 3.8$ & $47.7 \pm 5.3$ & $\mathrm{ND}$ \\
9 & $34.8 \pm 4.7$ & $57.2 \pm 4.3$ & $\mathrm{ND}$ \\
10 & $39.3 \pm 4.2$ & $69.7 \pm 4.9$ & $\mathrm{ND}$ \\
11 & $43.5 \pm 4.6$ & $78.4 \pm 3.8$ & $\mathrm{ND}$ \\
\hline
\end{tabular}

${ }^{1}$ Values are means of 3 replications \pm SD.

${ }^{2} \mathrm{ND}=$ none detected. 


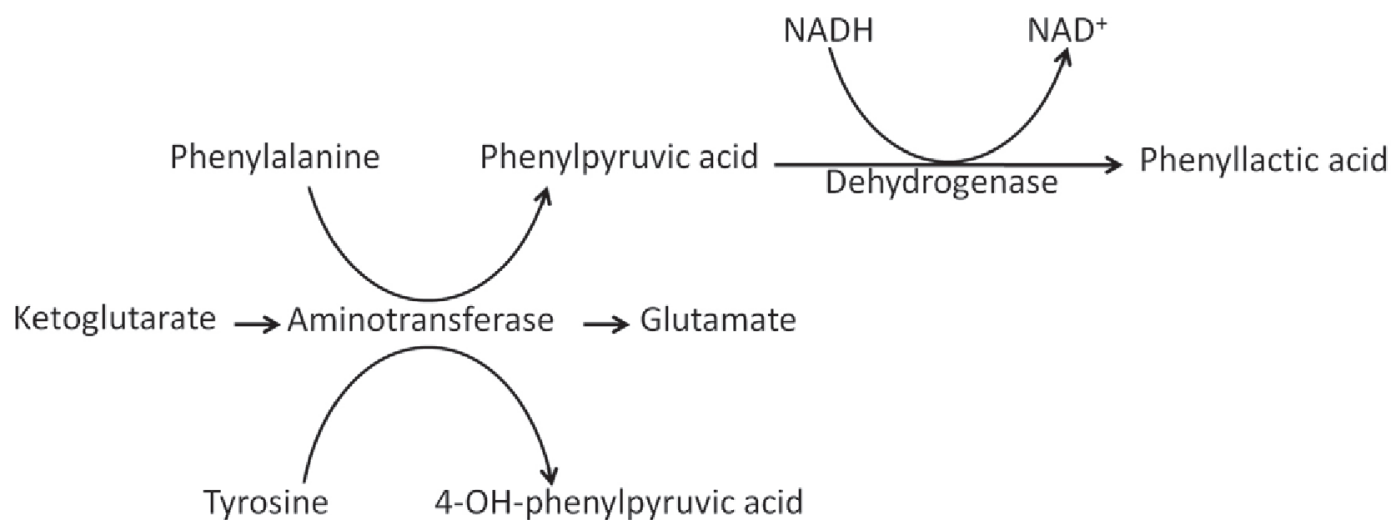

Figure 2. Possible pathway of 3-phenyllactic acid (PLA) production by Pediococcus pentosaceus SK25 in fermented milk.

mentation process, and the PLA concentration reached $82.5 \mathrm{mg} / \mathrm{L}$ after fermentation for $12 \mathrm{~h}$; in contrast, PLA biosynthesis was almost completely inhibited by the tyrosine supplement (Table 2).

The PLA biosynthesis pathway in LAB is well characterized (Mu et al., 2012b). 3-Phenyllactic acid production is a side pathway of phenylalanine metabolism, in which phenylalanine is transaminated to phenylpyruvic acid by aminotransferase and phenylpyruvic acid is further reduced to PLA by a dehydrogenase (Figure 2). Phenylalanine supplementation may thus increase PLA biosynthesis. Conversely, the transamination reaction of phenylalanine is catalyzed by aromatic aminotransferase, which has a broad substrate spectrum, including tyrosine (Yvon et al., 1997). Therefore, tyrosine inhibits the transamination of phenylalanine and tyrosine supplementation thus decreases PLA production. Many studies have shown that tyrosine supplementation strongly inhibits PLA production, but it did not completely inhibit the PLA production of LAB strains in flask fermentation (Valerio et al., 2004; Mu et al., 2012a). In our experiment, PLA production was almost completely inhibited within $12 \mathrm{~h}$ by tyrosine supplementation during $P$. pentosaceus SK25 fermentation in milk.

In conclusion, PLA was produced in milk by $P$. pentosaceus SK25 during laboratory fermentation process, and the PLA production level was increased by supplementation with phenylalanine. Our study presents a possibility that using PLA-producing LAB strains as starters may produce PLA in LAB-fermented milk products to improve antimicrobial activity against food-borne pathogenic bacteria and food-spoiling fungi.

\section{ACKNOWLEDGMENTS}

This work was supported by the Major State Basic Research Development Program of China (973
Program, No. 2012CB720802) and the State HighTech Development Plan of China (863 Program, No. 2011AA100904).

\section{REFERENCES}

Dallagnol, A. M., C. A. N. Catalan, M. I. Mercado, G. F. De Valdez, and G. C. Rollan. 2011. Effect of biosynthetic intermediates and citrate on the phenyllactic and hydroxyphenyllactic acids production by Lactobacillus plantarum CRL 778. J. Appl. Microbiol. 111:1447-1455.

Dallagnol, A. M., M. Pescuma, G. F. De Valdez, and G. C. Rollan. 2013. Fermentation of quinoa and wheat slurries by Lactobacillus plantarum CRL 778: proteolytic activity. Appl. Microbiol. Biotechnol. 97:3129-3140.

Dieuleveux, V., and M. Gueguen. 1998. Antimicrobial effects of D3-phenyllactic acid on Listeria monocytogenes in TSB-YE medium, milk, and cheese. J. Food Prot. 61:1281-1285.

Dimitrova, B., R. Gevrenova, and E. Anklam. 2007. Analysis of phenolic acids in honeys of different floral origin by solid-phase extraction and high-performance liquid chromatography. Phytochem. Anal. 18:24-32.

Gerez, C. L., M. S. Carbajo, G. Rollan, G. Torres Leal, and G. F. De Valdez. 2010. Inhibition of citrus fungal pathogens by using lactic acid bacteria. J. Food Sci. 75:M354-359.

Lavermicocca, P., F. Valerio, A. Evidente, S. Lazzaroni, A. Corsetti, and M. Gobbetti. 2000. Purification and characterization of novel antifungal compounds from the sourdough Lactobacillus plantarum strain 21B. Appl. Environ. Microbiol. 66:4084-4090.

Lavermicocca, P., F. Valerio, and A. Visconti. 2003. Antifungal activity of phenyllactic acid against molds isolated from bakery products. Appl. Environ. Microbiol. 69:634-640.

Li, X., B. Jiang, and B. Pan. 2007. Biotransformation of phenylpyruvic acid to phenyllactic acid by growing and resting cells of a Lactobacillus spp. Biotechnol. Lett. 29:593-597.

Mu, W., S. Yu, L. Zhu, B. Jiang, and T. Zhang. 2012a. Production of 3-phenyllactic acid and 4-hydroxyphenyllactic acid by Pediococcus acidilactici DSM 20284 fermentation. Eur. Food Res. Technol. 235:581-585.

Mu, W., S. Yu, L. Zhu, T. Zhang, and B. Jiang. 2012b. Recent research on 3-phenyllactic acid, a broad-spectrum antimicrobial compound. Appl. Microbiol. Biotechnol. 95:1155-1163.

Ndagano, D., T. Lamoureux, C. Dortu, S. Vandermoten, and P. Thonart. 2011. Antifungal activity of 2 lactic acid bacteria of the Weissella genus isolated from food. J. Food Sci. 76:M305-311.

Ohhira, I., S. Kuwaki, H. Morita, T. Suzuki, S. Tomita, S. Hisamatsu, S. Sonoki, and S. Shinoda. 2004. Identification of 3-phenyllactic acid as a possible antibacterial substance produced by Enterococcus faecalis TH10. Biocontrol Sci. 9:77-81. 
Rodriguez, N., J. M. Salgado, S. Cortes, and J. M. Dominguez. 2012. Antimicrobial activity of D-3-phenyllactic acid produced by fedbatch process against Salmonella enterica. Food Contr. 25:274284.

Ryan, L. A. M., F. Dal Bello, M. Czerny, P. Koehler, and E. K. Arendt. 2009. Quantification of phenyllactic acid in wheat sourdough using high resolution gas chromatography-mass spectrometry. J. Agric. Food Chem. 57:1060-1064.

Schwenninger, S. M., C. Lacroix, S. Truttmann, C. Jans, C. Sporndli, L. Bigler, and L. Meile. 2008. Characterization of low-molecularweight antiyeast metabolites produced by a food-protective Lactobacillus-Propionibacterium coculture. J. Food Prot. 71:2481-2487.

Somkuti, G. A., and D. H. Steinberg. 2010. Pediocin production in milk by Pediococcus acidilactici in co-culture with Streptococcus thermophilus and Lactobacillus delbrueckii ssp. bulgaricus. J. Ind. Microbiol. Biotechnol. 37:65-69.

Tanasupawat, S., S. Okada, M. Kozaki, and K. Komagata. 1993. Characterization of Pediococcus pentosaceus and Pediococcus acidilacti$c i$ strains and replacement of the type strain of $P$. acidilactici with the proposed neotype DSM 20284. Request for an opinion. Int. J. Syst. Bacteriol. 43:860-863.

Tuberoso, C. I., E. Bifulco, P. Caboni, G. Sarais, F. Cottiglia, and I. Floris. 2011. Lumichrome and phenyllactic acid as chemical markers of thistle (Galactites tomentosa Moench) honey. J. Agric. Food Chem. 59:364-369.

Valerio, F., P. Lavermicocca, M. Pascale, and A. Visconti. 2004. Production of phenyllactic acid by lactic acid bacteria: an approach to the selection of strains contributing to food quality and preservation. FEMS Microbiol. Lett. 233:289-295.

Van der Meulen, R. I. Scheirlinck, A. Van Schoor, G. Huys, M. Vancanneyt, P. Vandamme, and L. De Vuyst. 2007. Population dynamics and metabolite target analysis of lactic acid bacteria during laboratory fermentations of wheat and spelt sourdoughs. Appl. Environ. Microbiol. 73:4741-4750.

Yvon, M., S. Thirouin, L. Rijnen, D. Fromentier, and J. C. Gripon. 1997. An aminotransferase from Lactococcus lactis initiates conversion of amino acids to cheese flavor compounds. Appl. Environ. Microbiol. 63:414-419. 